\title{
A SELECTIVE PAGING SCHEME BASED ON ACTIVITY IN CELLULAR MOBILE NETWORKS FOR REPORTING CENTRE
}

\author{
Aakanksha Sharma ${ }^{1}$, Anurag Jain ${ }^{2}$ and Anubhav Sharma \\ ${ }^{1}$ C.S.E Department, Radharaman Institute of Technology and Science, Bhopal.(M.P) \\ aakanksha1006@gmail.com \\ ${ }^{2}$ Head Of Department C.S.E Department, Radharaman Institute of Technology and \\ Science, Bhopal.(M.P) \\ anurag.akjainegmail.comlegmail.com \\ ${ }^{3}$ Head Of Department C.S.E Department, Acropolis Institute Of Technology And \\ Research, Bhopal.(M.P) \\ anubhav_sharma0025@yahoo.com
}

ABSTRACT

The foremost aim of cellular mobile communication is to find out existing location of mobile terminals to give out the service, which is well-known as location management. The LM involves tracking of (mobile terminal's) MT's up-to-date location, which moves freely across different cells in order to provide them services. Every MT undergoes same number of updates when passes through a definite region. One such scheme is reporting centre in which, some of the cells are designated as reporting centres and all close by cells up to the next reporting centre belong to vicinity of same reporting centre. MT updates its location whenever it crosses vicinity of its current reporting centre, which happens no more than when it enters into another reporting centre and therefore a $L U$ is triggered. To deliver a call, network pages current reporting centre and its entire vicinity simultaneously to locate the target MT. We have applied predictionbased selective paging on reporting centre scheme in cellular mobile networks, which reduces paging cost without affecting the location update cost. Paging cost by the side of with LM cost for both the conventional and proposed schemes will be updated as a consequence which gives the results.

\section{KEYWORDS}

Cellular mobile network, reporting center, location management (LM), location update (LU), mobile terminals (MT), registration, paging.

\section{INTRODUCTION}

Nowadays cellular network is on the rise of becoming popular as a result of the more and more common network coverage and available mobile applications. A cellular network is a kind of radio network which is distributed over cells, and with all cells served by more than one base station or cell site. An overall cellular network contains a number of different elements from the base transceiver station (BTS) itself with its antenna back through a base station controller (BSC), and a mobile switching centre (MSC) to the location registers (HLR and VLR) and the link to the public switched telephone network (PSTN).

This research has been carried out with following major objectives:

$>$ To reduced the overhead involved in existing movement based dynamic LM scheme.

DOI : 10.5121/ijwmn.2013.5407 
To apply successive paging in reporting center scheme and analyze its effects on location management cost.

$>$ To develop an ACTIVITY BASED MODEL in paging scheme that can predict user location with high accuracy.

$>$ Evaluating the performance of proposed scheme(s) against existing scheme(s) with the help of mathematical modeling and/or simulation (using Java programming language).

\section{ObJeCtive AND Problem SPECIFICATION}

The idea behind successive paging comes from the fact that in bounded non reporting cell configuration, the vicinity of clusters can be divided into two or more groups of cells separated by a barrier of reporting cells. Vicinity of clustered reporting centers 1 and 6 can be separated into two different groups consisting of cells $0,4,5$ and cells 2, 3, 7 shown by dashed lines, the cluster members (cells 1 and 6) form a barrier that separate these groups. According to proposed scheme, instead of paging whole vicinity in once it should be done in following two steps:

Page all the cells in any one group and all cluster members simultaneously, if target MT is found then stop.

$>$ Otherwise page all cells in the other group simultaneously.

A solid line of barrier, known as the reporting cells form which means a user will have to enter one of the reporting cells to get to the other side. For example, in Fig.1 (b), cells 1, 5, 8, 9, 10, 11 and 15 are reporting centres. Vicinity of reporting centre 1 consists of cells $0,2,3,4,6,7$ and 1 itself. Likewise, vicinity of reporting centre 9 consists of cells $0,4,12,13,14$ and 9. An MT in cell 13 must cross a reporting centre to enter into cell 6 and therefore LU will be triggered. A cell may fall under vicinity of more than one reporting centres [11]. For illustration in fig.1 (b), cells 2, 3, 6 and 7 fall under the vicinity of reporting centres 1, 5, 10 and 11. That means whether the reporting centres of an MT is either cell 1, 5, 10 or 11, these four cells will for all time be paged whenever a call arrives of that MT. Under reporting centre scheme, non reporting cells may be bounded or unbounded [12]. Fig.1 (a) and (b) shows bounded and unbounded non reporting cell configurations respectively.

Here an activity based model have been made which gives a appropriate scheduled movements to MTs and have applied prediction-based selective paging on reporting centre scheme in cellular mobile networks, which reduces paging cost without moving the location update cost. Paging cost and LM cost for both the conventional and proposed schemes will be updated as a result [2] [12].

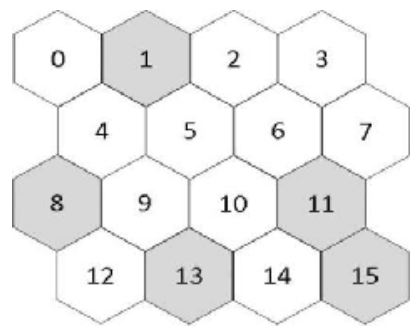

(a) Unenclosed non-reporting cell arrangement 


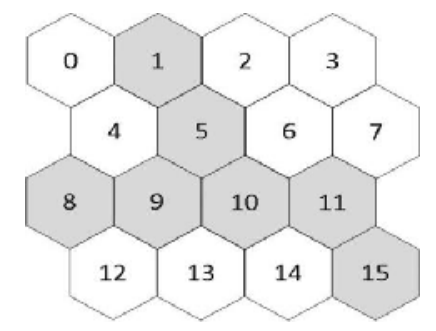

(b) Enclosed non-reporting cell arrangement

\subsection{Background Study}

Fig.1 Cell Configuration

A number of methodologies have been proposed for location management. Most of these schemes aim to reduce either location update cost or paging cost. Very a small number of them have reduced both location update cost and paging cost.

A location management scheme where with each LU message from a MS, a LA is distinct for the MS based on subscriber mobility history is. As long as a mobile moves within its present LA, no update message is triggered. Only when a mobile moves out of its present LA, a LU is triggered and a new LA is defined for the MS. This algorithm also considers speed and call entrance probability of the subscriber to define the LA size. Since this algorithm tries to reduce LM cost for individuals, it significantly outperforms static algorithms, but it requires more processing power as compared to stationary algorithms. This algorithm reduces this overhead at the cost of some added logic and memory in the MS and network [1].

An intelligent LM scheme by taking User Profile History to reduce the location update cost by combining Back-Propagation Algorithm and Cascaded Correlation Neural Network. This scheme reduces the LM cost significantly [2].

An algorithm to reduce paging cost using speed adaptive policy. It uses speed distribution and random walk model to develop look-up tables for mobile users. These tables are worn to find out subarea size to be paged. Paging area is further reduced by introduction of 120 degree paging zones. A paging agent was used to store previous VLR address and helps to find out optimal paging zone [3].

Authors propose a hybrid location update scheme in which, movement-based and time based location update approaches are combined for the improvement of QoS. This technique has two parts: first time then movement, and first movement then time. The effectiveness of the proposed scheme is checked with CMR, velocity and time. In Zheng, authors proposed an adaptive location update area design for wireless cellular network under 2D Markov walk model, and this scheme is helpful for LU area design [4].

Authors analysed the performance of various LM schemes such as one step pointer forwarding scheme, IS-41 and pointer forwarding techniques. It was experiential that each LM scheme has its own specialization, utilization and testability and it is based on CMR and threshold values [5].

A location management scheme using mobility in sequence of mobile users in wireless mobile networks. In this scheme, the location registration process caches location information of visited cells to reduce the duplicated registrations, the paging process exploits the zone of user movement patterns to predict the paging area and, therefore to reduce the paging cost in location explore procedure [8]. 
A application that based on an MT's movement model that captures mobility behaviour, the network can expect the location of MT at cell level because many trips of MTs follow routinely trajectories. If MT visits an expected cell, the target cell , before a certain movement threshold is reached, its movement counter is reset to zero. Otherwise the mobile terminal will trigger a location update message when the mentioned threshold is reached. This scheme reduces signalling traffic [9].

\subsection{Work done}

The activity-based mobility model is implemented using Java (jdk1.7) for simulation purpose [20].Work carried out in this research can be summarized as follows:

- We have applied prediction-based selective paging on reporting center scheme in cellular mobile networks, which reduces paging cost without affecting the location update cost.

\subsubsection{Activity Based Mobility Model}

Generally, MTs do not move across cells in fully random fashion. The vital principle is an individual's daily activity pattern, including such activities as going to work-place or school. In activity based mobility model, an MT moves to a particular cell at a particular time (following same or dissimilar paths each time).

\subsubsection{Simulation Setup}

Number of Cells $=49$

Number of MTs=100, Every MT is initially assigned a Home randomly

Number of Colleges $=5$

Number of Work Places $=5$

Number of Fitness centres $=4$

\subsubsection{Schedule Movements:}

6:00 AM Home to Fitness centre (Only FIT MTs)

7:00 AM Fitness centre to Home (Only FIT MTs)

8:00 AM Home to College (Only Students)

10:00 AM Home to Work place (Only Workers)

14:00 PM College to Home (Only Students)

17:00 PM Work place to Home (Only Workers)

Initially, every $M T$ will be assigned a home randomly. Every $M T$ will initially be placed at its home. There will be Fitness centres, work places, colleges and home assigned to MTs [9]. MTs will move from source to destination cell timely. Paging cost and LM cost for both the schemes will be updated accordingly.LU cost will remain same in both the schemes. Path between every source cell to destination cell will be determined at runtime. An MTMover thread will move each MT by one cell towards its destination until it reaches its current destination. On every move, it is checked that if the new cell is a reporting centre. If yes then if it is not the current reporting centre of the moved MT then an LU operation is performed by the MT. The Clock will rise the time 10seconds in each two seconds. Clock thread will invoke MTMover thread. The CallGenerator thread will keep initiating calls to randomly picked MT. Called MT will be paged according to VLR database entry. Its database at VLR level will be restructured if essential. Movement of MTs will be distinct as follow: 
- From 17 to 06 O'clock, stay at home

- At 06, leave for Fitness centre (Only fit MTs)

- At 07, leave for Home (Only fit MTs)

- At 08, leave for College (Only students)

- At 10, leave for Work place (Only Workers)

- At 14, leave for Home (Only Students)

- At 17, leave for Home (Only Workers)

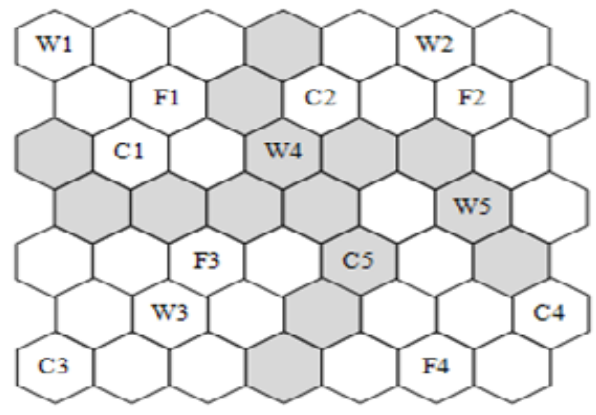

Fig.2 (a) an activity based illustration

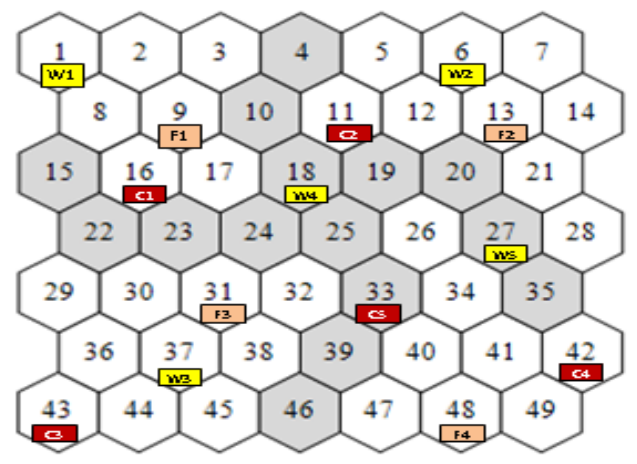

Fig.2 (b) an activity based illustration with assigned spaces

This time table will be followed for one week and results will be analysed.

At any moment, possible move from a cell (shown in bold) can be determined from following 2D-array:

Table 1. Movements of MT's

\begin{tabular}{|c|c|c|c|c|c|c|c|c|}
\cline { 2 - 10 } \multicolumn{1}{c|}{ Network } & 0 & 1 & 2 & 3 & 4 & 5 & 6 & 7 \\
\hline $\boldsymbol{0}$ & 0 & 0 & 0 & 0 & 0 & 0 & 0 & 0 \\
\hline $\mathbf{1}$ (Odd) & 0 & 1 & 2 & 3 & 4 & 5 & 6 & 7 \\
\hline 2 (Even) & 0 & 8 & 9 & 10 & 11 & 12 & $\mathbf{1 3}$ & 14 \\
& & & & & & & & \\
\hline
\end{tabular}


International Journal of Wireless \& Mobile Networks (IJWMN) Vol. 5, No. 4, August 2013

\begin{tabular}{|c|c|c|c|c|c|c|c|c|}
\hline $3($ Odd $)$ & 0 & 15 & 16 & 17 & 18 & 19 & 20 & 21 \\
\hline 4 (Even) & 0 & 22 & 23 & 24 & 25 & 26 & 27 & 28 \\
\hline $5($ Odd $)$ & 0 & 29 & 30 & 31 & 32 & 33 & 34 & 35 \\
\hline 6(Even) & 0 & 36 & 37 & 38 & 39 & 40 & 41 & 42 \\
\hline $7($ Odd $)$ & 0 & 43 & 44 & 45 & 46 & 47 & 48 & 49 \\
\hline
\end{tabular}

Note: Remember that first row and first column i.e. Oth row and 0th column are not used at all.

\section{CAlCulations}

\subsection{Location Management Cost}

A number of LM schemes have been proposed by various researchers so there must be some structure that can be second-hand to symmetry one scheme by means of the other. LM cost function comprises of two main components: updating cost and paging cost. Updating cost is the cost due to location update performed by MTs in the network whereas paging cost is caused by the network during location inquiries while locating an MT [1]. There are some other parameters that influence the total LM cost such as cost of database management in LU operation, cost in terms of wired line (backbone) network bandwidth used (that connects base stations to each other). Mostly these costs are assumed to be constant for all LM schemes. So combination of LU and paging cost are measured to be sufficient to compare different LM schemes.

$$
\text { Total LM Cost }=(\mathrm{C} \times \mathrm{NLU})+(\mathrm{NP})
$$

Where NLU denotes the number of location updates performed during simulation time T, NP denotes the number of paging operations performed during time $\mathrm{T}$ and $\mathrm{C}$ is a constant, which is the ratio of single LU cost to the cost of paging solo cell. It is said that the cost of a LU is ten times the cost of a paging operation. In light of this fact, we used $\mathrm{C}=10$.

Reporting center scheme decreases LU cost but increases the paging cost. In unbounded nonreporting cell configuration, since the vicinity of reporting centers are not bounded therefore paging cost may enlarge noticeably as compared to bounded non-reporting cell configuration [14]. This paper presents a simple norm for clustering reporting centers in bordered non reporting cell configuration that decreases total LM cost but since clustering always increases the paging cost therefore a successive paging scheme is proposed which, when combined with clustering technique, curtails both updating cost and paging cost resultant into remarkable reduction in total LM cost.

\subsection{Paging Cost}

In attempt to locate target MT as quickly as possible, multiple methods of paging have been proposed by the researchers. The most basic method used is Simultaneous paging, where every cell in the MT's LA is paged at the same time [7] [9]. If LA contains large number of cells, this scheme costs terribly high. In Sequential Paging, cells within an LA are paged one after the other, in order of decreasing user dwelling possibility. If the user resides in an infrequently occupied 
location, long delay may occur in finding the MT. However, this scheme has too much computational overhead incurred through updating and maintaining the matrix [19].

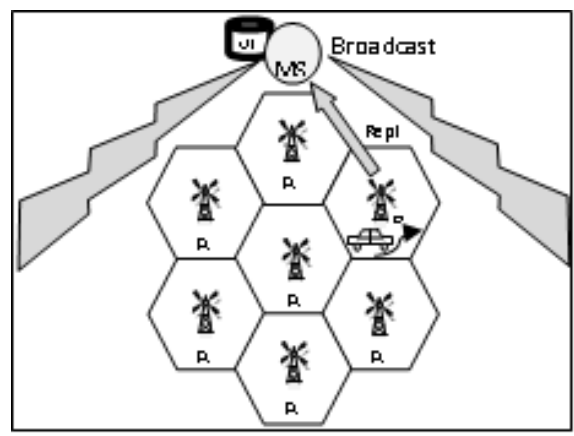

Fig.3 Paging Method

Determine row number of source/destination cell:

Let us say source node is 13 and destination cell is 37 (i.e. W3), then

Source cell: $\quad \operatorname{ceil}(13 / 7)=2$

Destination cell: $\quad \operatorname{ceil}(37 / 7)=6$

Determine column number of source/destination cell:

Let us say source node is 13 , then

Source cell:

$$
\begin{array}{ll} 
& \operatorname{ceil}(13 / 7)=2 \\
\Rightarrow & 7 *(2-1)=7 \\
\Rightarrow & 13-7=6
\end{array}
$$

Destination cell:

$$
\begin{aligned}
& \operatorname{ceil}(37 / 7)=6 \\
& \Rightarrow \quad 7 *(6-1)=35 \\
& \Rightarrow \quad 37-35=\mathbf{2}
\end{aligned}
$$

Thus row and col. no. of source cell are $2 \& 6$ i.e. index is $[2,6]$

And row and col. no. of destination cell are $\quad 6 \& 2$ i.e. index is $[6,2]$

- If $x$ co-ordinates of source \& destination are same then-

Move vertically, one cell each time so that difference between $\mathrm{x}$ reduces every time by 1

The variable sign $=$ '+' or '-' tells whether to decrement or increment at every step

- If $y$ co-ordinates of source \& destination are same then-

Move vertically, one cell each time so that difference between y reduces every time by 1

The variable sign = '+' or '-' tells whether to decrement or increment at every step

- If both remain same then-

Go no where 
- If both change then-

assign values to xsign and ysign where

xsign $=$ ' + ' or '-' tells $x$ field of MT needs to be incremented or decremented ysign $=$ ' + ' or '-' tells $y$ field of MT needs to be incremented or decremented Now invoke the move_generator method:

while $\mathrm{x}_{\text {source }}$ and $\mathrm{y}_{\text {source }}$ become equal to $\mathrm{x}_{\text {destination }}$ and $\mathrm{y}_{\text {destination }}$ do as follow:

If row number is odd then

If $x$ sign $=+$ and ysign $=+$ then Best move will be cell[ $[x+1, y]$

If xsign $=-$ and ysign $=+$ then Best move will be cell $[x-1, y]$

If $x$ sign $=+$ and ysign $=-$ then Best move will be cell[ $[x+1, y-1]$

If xsign $=-$ and ysign $=-$ then Best move will be cell[ $x-1, y-1]$

If row number is even then

If $x$ sign $=+$ and ysign $=+$ then Best move will be cell $[x+1, y+1]$

If xsign $=-$ and ysign $=+$ then Best move will be cell[ $x-1, y+1]$

If xsign $=+$ and ysign $=-$ then Best move will be cell $[x+1, y]$

If xsign=- and ysign=- then Best move will be cell[ $[x-1, y]$

When to perform LU operation:

Arrays used:

Following array contains the IDs of all the reporting cells:

Rep_Cntr_IDs[] $=\{4,10,15,18,19,20,22,23,24,25,27,33,35,39,46\}$;

WorkPlace []$=\{0,1,6,37,18,27\}$;

It means W1 is located in cell-1 i.e. cell whose cell-id is 1, W2 is located in cell-6, W3 is located in cell-37, W4 is located in cell 18 and W5 is located in cell-27. The $0^{\text {th }}$ index is not used at all so that cell-id of W3 can be determined simply as WorkPlace [3]

Similarly other arrays are:

College []$=\{0,16,11,43,42,33\}$

FitnessCenter []$=\{0,9,13,31,48\}$

This is achieved by paging process in which, the MSC broadcasts polling signals to all cells contained by the LA of the called MT.

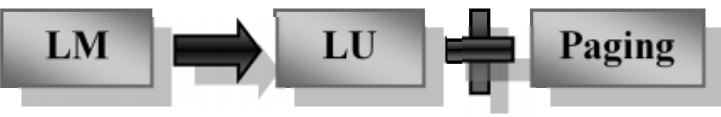


International Journal of Wireless \& Mobile Networks (IJWMN) Vol. 5, No. 4, August 2013

3.3.Studying the Effects of Selective Paging on Reporting Centre Scheme Using An Activity Based Model

\subsubsection{Comparison of Paging Cost}

Table 1. Cumulative Paging Cost involved during the week

\begin{tabular}{|c|c|c|}
\hline Till Day-No. & $\begin{array}{c}\text { Conventional } \\
\text { scheme }\end{array}$ & $\begin{array}{c}\text { Proposed } \\
\text { scheme }\end{array}$ \\
\hline Till Day-1 & 12690 & 10929 \\
\hline Till Day-2 & 25449 & 19669 \\
\hline Till Day-3 & 38237 & 25898 \\
\hline Till Day-4 & 50991 & 30860 \\
\hline Till Day-5 & 63644 & 34510 \\
\hline Till Day-6 & 76399 & 37926 \\
\hline Till Day-7 & 89188 & 40797 \\
\hline
\end{tabular}

Graphical observation-

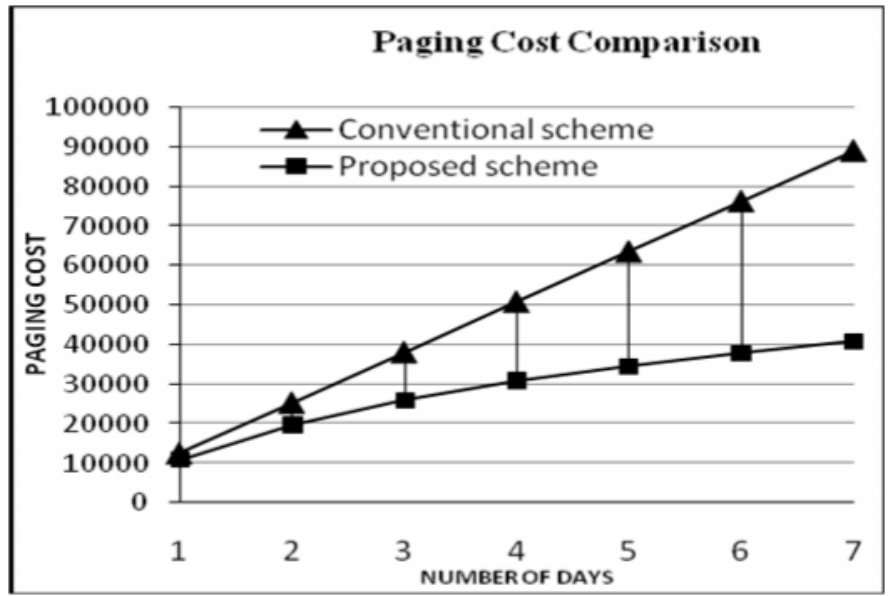

\subsubsection{Comparison of Location Management Cost-}

Table 2. Cumulative Location Management Cost

\begin{tabular}{|c|c|c|}
\hline $\begin{array}{c}\text { Till Day- } \\
\text { No. }\end{array}$ & Conventional scheme & Proposed scheme \\
\hline Till Day-1 & 560802190 & 560800429 \\
\hline Till Day-2 & 1114202909 & 1114197129 \\
\hline Till Day-3 & 1703612697 & 1703600358 \\
\hline Till Day-4 & 2282936751 & 2282916620 \\
\hline Till Day-5 & 2869448924 & 2869419790 \\
\hline Till Day-6 & 3452300879 & 3452262406 \\
\hline Till Day-7 & 4009871298 & 4009822907 \\
\hline
\end{tabular}


Graphical observation-

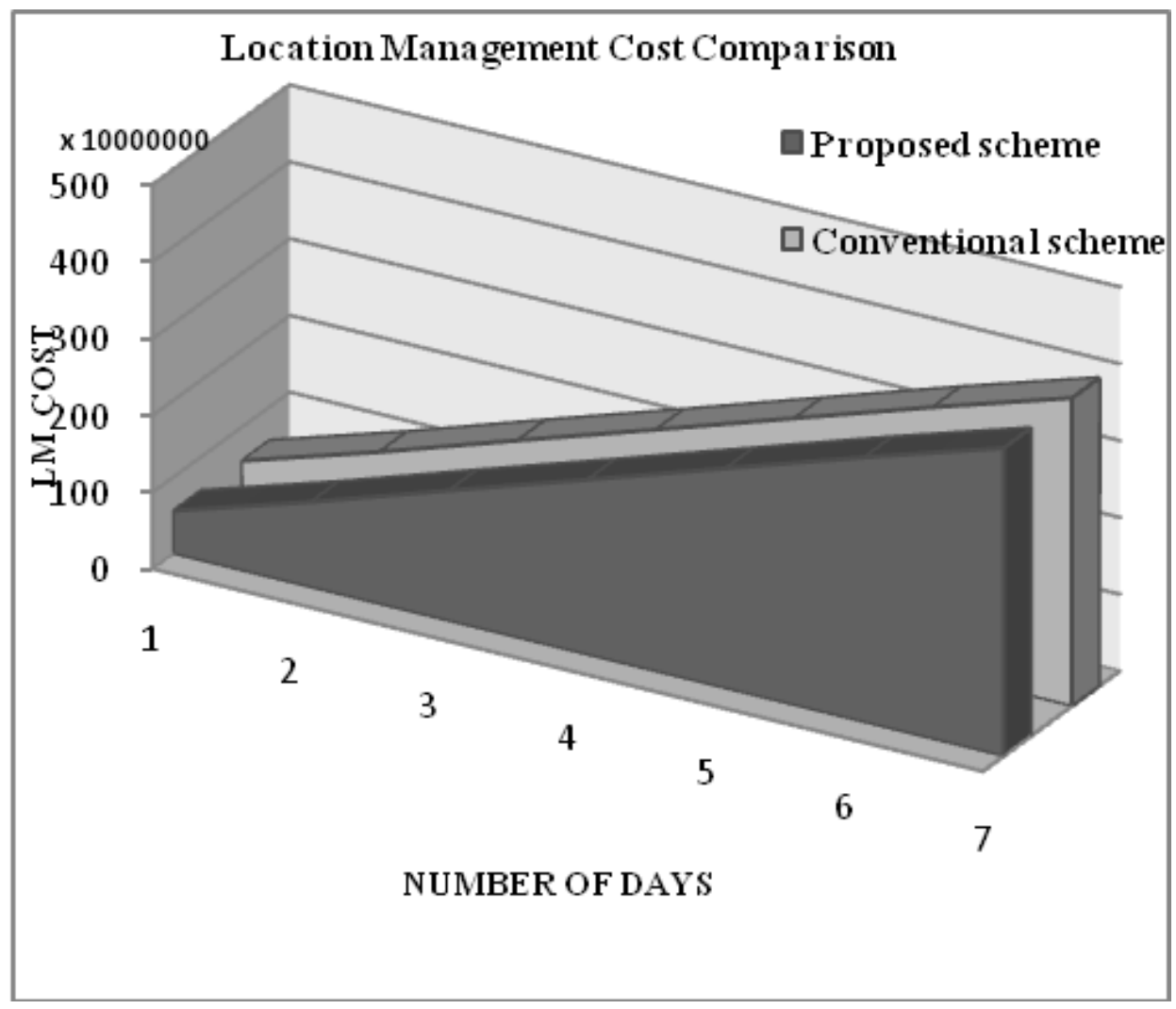

\subsection{RESULTS}

All the results when number of MTs are 100-

Cumulative statistics till the end of Day-1

Number of calls generated: 716

Number of page Faults: 611

Paging cost in conventional scheme: 12690

Paging cost in proposed scheme: 10929

LU cost: 560789500

LM cost in conventional scheme: 560802190

LM cost in proposed scheme: 560800429

\section{Cumulative statistics till the end of Day-2}

Number of calls generated: 1436

Number of page Faults: 1089

Paging cost in conventional scheme: 25449

Paging cost in proposed scheme: 19669

LU cost: 1114177460

LM cost in conventional scheme: 1114202909 
LM cost in proposed scheme: 1114197129

\section{Cumulative statistics till the end of Day-3}

Number of calls generated: 2156

Number of page Faults: 1418

Paging cost in conventional scheme: 38237

Paging cost in proposed scheme: 25898

LU cost: 1703574460

LM cost in conventional scheme: 1703612697

LM cost in proposed scheme: 1703600358

\section{Cumulative statistics till the end of Day-4}

Number of calls generated: 2877

Number of page Faults: 1674

Paging cost in conventional scheme: 50991

Paging cost in proposed scheme: 30860

LU cost: 2282885760

LM cost in conventional scheme: 2282936751

LM cost in proposed scheme: 2282916620

\section{Cumulative statistics till the end of Day-5}

Number of calls generated: 3596

Number of page Faults: 1852

Paging cost in conventional scheme: 63644

Paging cost in proposed scheme: 34510

LU cost: 2869385280

LM cost in conventional scheme: 2869448924

LM cost in proposed scheme: 2869419790

\section{Cumulative statistics till the end of Day-6}

Number of calls generated: 4316

Number of page Faults: 2012

Paging cost in conventional scheme: 76399

Paging cost in proposed scheme: 37926

LU cost: 3452224480

LM cost in conventional scheme: 3452300879

LM cost in proposed scheme: 3452262406

\section{Cumulative statistics till the end of Day-7}

Number of calls generated: 5037

Number of page Faults: 2140

Paging cost in conventional scheme: 89188

Paging cost in proposed scheme: 40797

LU cost: 4009782110

LM cost in conventional scheme: 4009871298

LM cost in proposed scheme: 4009822 
Table 3. Location management cost with conventional and proposed schemes, when no. of MTs are $40,60,80,100,130$ and 200

\begin{tabular}{|c|c|c|c|c|c|c|c|}
\hline DAYS & No. of MTs & 40 & 60 & 80 & 100 & 130 & 200 \\
\hline \multirow{2}{*}{ DAY 1} & CONVENTIONAL & 224320876 & 336481314 & 448641752 & 560802190 & 729042847 & 1121604380 \\
\hline & PROPOSED & 224320171.6 & 336480257.4 & 448640343.2 & 560800429 & 7290405577 & 1121600858 \\
\hline \multirow{2}{*}{ DAY 2} & CONVENTIONAL & 445681163.6 & 6684721745 & 8912962327 & 1114202909 & 1448463782 & 2228405818 \\
\hline & PROPOSED & 445678851.6 & 668518277.4 & 891357703.2 & 1114197129 & 1448456268 & 2228394258 \\
\hline \multirow{2}{*}{ DAY 3} & CONVENTIONAL & 681445078.8 & 1022167618 & 1362890158 & 1703612697 & 2214696506 & 3407225394 \\
\hline & PROPOSED & 681440143.2 & 1022160215 & 1362880286 & 1703600358 & 225680465.4 & 3407200716 \\
\hline \multirow{2}{*}{ DAY 4} & CONVENTIONAL & 913175900.4 & 1369762051 & 1826349401 & 2282936751 & 2967817776 & 4565873502 \\
\hline & PROPOSED & 913166648 & 1369749972 & 1826333296 & 2282916620 & 2967791606 & 4565833240 \\
\hline \multirow{2}{*}{ DAY 5} & CONVENTIONAL & 1147779570 & 1721669354 & 2295559139 & 2869448924 & 3730283601 & 5738897848 \\
\hline & PROPOSED & 1147767916 & 1721651874 & 2295535832 & 2869419790 & 3730245727 & 5738839580 \\
\hline \multirow{2}{*}{ DAY 6} & CONVENTIONAL & 1380920352 & 2071380527 & 2761840703 & 3452300879 & 4487991143 & 6904601768 \\
\hline & PROPOSED & 1380904962 & 2071357444 & 2761809925 & 3462292406 & 4487941128 & 6904524812 \\
\hline \multirow[t]{2}{*}{ DAY 7} & CONVENTIONAL & 1603948519 & 2405922779 & 3207897038 & 4009871298 & 5212832687 & 8019742596 \\
\hline & PROPOSED & 1603929162 & 2405863744 & 3207558325 & 4009822907 & 5212769779 & 8019645814 \\
\hline
\end{tabular}

\section{CONClusions}

The key issues in cellular mobile communication is to find current location of mobile terminals to deliver the service, which is known as location management (LM).The LM involves tracking of (mobile terminal's) MT's up-to-date location, who move freely across different cells in order to provide them services. We have applied prediction-based selective paging on reporting center scheme in cellular mobile networks, which reduces paging cost without affecting the location update cost. Paging cost and LM cost for both the conventional and proposed schemes update accordingly. In activity based mobility model, an MT moves towards a destination cell at a specific time (following same or different paths each time). Hence results reveal that the proposed scheme predicts user location with high accuracy and reduces paging cost remarkably.

\section{Future Work}

Reporting center scheme selects some cells as reporting cells thus changes the paging cost and updating cost of the wireless network. The motivation of the variation is that it will decrease updating cost as users move between two reporting centers. In order to see whether this result is statistically significant, the query rate and mobility rate could use other sets of values in the simulation. Currently we have applied the proposed scheme only on daily worker mobility model. In future we can apply it on various other mobility models to analyze the variations in performance of proposed scheme.

\section{ACKNOWLEDGEMENTS}

I pay gratitude to supreme power "The Almighty", who blessed me with love, patience and vigor to complete this paper successfully. I would like to express my sincere thanks to my guide Prof. Anurag Jain, HOD, Department of Computer Science \& Engineering, R.I.T.S for his 
wisdom and abundance of encouragements from the very early stage of this research, as well as giving me constant support throughout the dissertation process and I also give my special thanks to Dr. A. K. Sachan (Director, RITS) for his great support and motivating me to complete my work. I give special thanks to Mr. Vijendra Singh Bhadauria, who always being willing to help me finding solutions to my problems. His valuable advice and support, was an inspiration and driving force for me.

\section{REFERENCES}

[1] Ajit Pal and Digvijay Singh Khati, "Dynamic location management with variable size location areas", IEEE, International Conference on Computer Networks and Mobile Computing, Oct. 2001, pp. 73-78.

[2] Ashish Goel, Navankur Gupta and Prakhar Kumar, "A Speed Based Adaptive Algorithm for Reducing Paging Cost in Cellular Networks", 2nd IEEE International Conference on

[3] Computer Science and Information Technology, 2009, pp. 22-25.

[4] C. Lee, C. H. Ke and C. C. Chen, "Improving location management for mobile users with frequently visited locations", Elsevier, Performance Evaluation, Vol. 43, 2001, pp. 15-38.

[5] D. W. Huang, P. Lin and C. H. Gan, "Design and performance study for a mobility management mechanism (WMM) using location cache for wireless mesh networks", IEEE Transaction on Mobile Computing, 2008, pp. 546-556.

[6] Amar Pratap Singh and M. Karnan, "Intelligent Location Management using Soft Computing Technique", IEEE, 2nd International Conference on Communication Software and Networks, 2010, pp. 343-346.

[7] Vicente Casares-Giner and Pablo Garcia-Escalle, "A lookahead strategy for movement based location update in wireless cellular networks", IEEE, 6th International Conference On Information Technology: New Generations, 2009, pp. 1171-1177

[8] Kenneth W. Shum and Chi Wan Sung, "Kalman-filter based predictive location management for PCS networks", The 57th IEEE Semiannual Vehicular Technology Conference, Apr. 2003, pp. 2701-2705.

[9] Karunaharan Ratnam, Sampath Rangarajan and Anton T. Dahbura, "An efficient fault tolerant location management protocol for personal communication networks", IEEE Trans. Vehicular Technology, Nov. 2000, pp. 2359-2369.

[10] Sun-Jin Oh, "The location management scheme using mobility information of mobile users in wireless mobile networks", IEEE International Conference on Computer Networks and Mobile Computing, Oct. 2003, pp. 230-237.

[11] Vijendra S. Bhadauria, Ravindra Patel, Sanjeev Sharma, "Effects of Clustering and Successive Paging on Reporting Center Scheme: A Cost Analysis" International Journal of Computer Applications (0975 - 8887) Volume 37- No.5, January 2012

[12 Vijendra S. Bhadauria, Ravindra Patel, Sanjeev Sharma, "A Comparative Study of Location Management Schemes: Challenges and Guidelines", Vijendra Singh Bhadauria et al. / International Journal on Computer Science and Engineering (IJCSE)

\section{[Reference Books]}

[13] A. Ghosh, J. Zhang, J. G. Andrews and R. Muhamed, "Fundamentals of LTE", 1st edition, ISBN-10: 0137033117, Prentice Hall.

[14] A. Mukherjee, S. Bandyopadhyay and D. Saha, "Location Management and Routing in Mobile Wireless Networks", ISBN- 1-58053-355-8, Artech House.

[15] K. Pahlavan and A. H. Levesque, "Wireless Information Networks", 2nd edition, ISBN-13: 978-0471-72542-8, Wiley.

[Web Links]

[16] http://awarenetworks.com/publications/6.108.10.CellularTelephony.pdf

[17] http://creativentechno.wordpress.com/2012/01/03/the-generations-1g-2g-3g-4g/

[18] http://media.johnwiley.com.au/product_data/excerpt/28/04714190/0471419028.pdf

[19] http://www.electronicsforu.com/EFYLinux/efyhome/cover/jun2003/Mobile-tech.pdf

[20] http://onlinelibrary.wiley.com/doi/10.1002/0471224561.ch2/summary

[21] http://www.antd.nist.gov/wctg/adhocvideo/html/ hybrid.htm 